ISSN 0103-9954

\title{
MIMOSOIDEAE (FABACEAE) DA REGIÃO DO MANSO, ANTÔNIO DIAS, MINAS GERAIS: DISTRIBUIÇÃO GEOGRÁFICA E SIMILARIDADE FLORÍSTICA NA FLORESTA ATLÂNTICA
}

\author{
MIMOSOIDEAE (FABACEAE) OF MANSO'S REGION, ANTÔNIO DIAS, MINAS GERAIS STATE: \\ GEOGRAPHIC DISTRIBUTION AND FLORISTIC SIMILARITY IN THE ATLANTIC FOREST
}

\author{
Ariane Cristine Araújo Silva ${ }^{1}$ Sânzia Romanova Duarte Ferreira da Silva Nunes ${ }^{2}$ \\ Sebastião Venâncio Martins ${ }^{3}$ Aurino Miranda Neto ${ }^{4}$
}

\begin{abstract}
RESUMO
A Região do Manso, localizada a 785 m de altitude no município de Antônio Dias, leste de Minas Gerais (1941'15"S, 42 48'45"W), está no domínio Atlântico na formação da floresta estacional semidecídua montana. No inventário das Mimosoideae ocorrentes na região, foram encontrados nove táxons que neste trabalho são analisados quanto aos limites de distribuição geográfica e somente os arbóreos são avaliados como indicadores das relações florísticas entre a região do Manso e outras áreas de Floresta Atlântica do Sudeste brasileiro. São eles: Senegalia martiusiana (Steud.) Seigler \& Ebingler, Anadenanthera colubrina (Vell.) Brenan var. cebil (Griseb.) Altschul, Anadenanthera peregrina (L.) Speg, Inga vera subsp. affinis (DC.) T.D. Penn., Mimosa debilis Humb. \& Bonpl. ex Willd. var. debilis, Mimosa pellita Humb. \& Bonpl. ex Willd. var. pellita, Piptadenia gonoacantha (Mart.) J.F. Macbr., Plathymenia reticulata Benth. e Stryphnodendron polyphyllum Mart. Cinco padrões de distribuição geográfica foram estabelecidos: 1. Anfiatlântico (1sp.); 2. Neotropical (1sp.); 3. América do Sul Ocidental-Centro-Oriental (4spp.); 4. Brasil Centro-Oriental (2spp.); 5. Brasil Atlântico Nordeste-Sudeste (1sp.). Os táxons quanto à preferência de habitat, foram tratados como elementos florísticos generalistas $(100 \%)$ e especialistas $(0 \%)$ do domínio Atlântico. Os resultados mostram maior similaridade entre o Manso e trechos de floresta situados nas altas elevações. Existem similaridades florísticas entre as florestas ombrófila densa e estacionais semidecíduas do sudeste, apoiando a proposição de origem comum dessas fitofisionomias.
\end{abstract}

Palavras-chave: fitogeografia; florística; floresta semidecídua; floresta atlântica.

\begin{abstract}
Manso's region is located at 785m of altitude in Antônio Dias town, east of Minas Gerais State, Brazil, which is in the Atlantic domain in a formation of Seasonal Semideciduous Montane Forest. In the inventory of Mimosoideae of that region, it wase found nine taxa which are analyzed regarding the limits of geographic distribution and only the arboreal are evaluated as floristic relation indicators between the region of Manso and other areas of southeastern Brazilian Atlantic forest. They are: Senegalia martiusiana (Steud.) Seigler \& Ebingler, Anadenanthera colubrina (Vell.) Brenan var. cebil (Griseb.) Altschul, Anadenanthera peregrina (L.) Speg, Inga vera subsp. affinis (DC.) T.D. Penn., Mimosa debilis Humb. \& Bonpl. ex Willd. var. debilis, Mimosa pellita Humb. \& Bonpl. ex Willd. var. pellita, Piptadenia gonoacantha (Mart.) J.F. Macbr., Plathymenia reticulata Benth. and Stryphnodendron polyphyllum Mart. Five geographical distribution

1 Bióloga, Mestre em Ciência Florestal, Universidade Federal de Viçosa, Av. PH Rolfs, s/n, CEP 36570-900, Viçosa (MG), Brasil. ariane.florestal@yahoo.com.br

2 Bióloga, Mestre em Botânica, Centro Universitário do Leste de Minas Gerais, Área da Saúde, Área da Saúde, Rua Bárbara Heliodora, 725, CEP 35160-215, Ipatinga (MG), Brasil. sanzia@universalisconsultoria.com.br

3 Engenheiro Florestal, Dr., Professor do Departamento de Engenharia Florestal, Universidade Federal de Viçosa, Av. PH Rolfs, s/n, CEP 36570-900, Viçosa (MG), Brasil. venancio@ufv.br

4 Engenheiro Florestal, MSc., Doutorando em Ciência Florestal, Laboratório de Restauração Florestal, Universidade Federal de Viçosa, Av. PH Rolfs, s/n, CEP 36570-900, Viçosa (MG), Brasil. aur.neto@gmail.com
\end{abstract}

Recebido para publicação em 30/03/2009 e aceito em 6/01/2015

Ci. Fl., v. 26, n. 4, out.-dez., 2016 
standards had been established: 1. Anfiatlântico (1sp.); 2. Neotropical (1sp.); 3. South America OccidentalCenter-Oriental (4spp.); 4. Brazil Center-Oriental (2spp.); 5. Atlantic Brazil Northeast-Southeast (1sp.). The taxa according to their preference habitat were labelled as floristic general elements $(100 \%)$ and specialist elements $(0 \%)$ of the Atlantic domain. The results show greater similarity between Manso and the forest stretches located in high elevations. There are floristic similarities between the dense mountain rain and seasonal semideciduous southeastern forest, supporting the proposal of common origin of these phytophysiognomies.

Keywords: phytogeograph; floristic; semideciduous forest; Atlantic forest.

\section{INTRODUÇÃO}

Leguminosae (Fabaceae) possui distribuição cosmopolita, incluindo cerca de 727 gêneros e 19.325 espécies, representando uma das maiores famílias de Angiospermas e também uma das principais do ponto de vista econômico (LEWIS et al., 2005). No Brasil, ocorrem cerca de 188 gêneros e 2.100 espécies, cuja ocorrência é muito significativa na maioria dos tipos vegetacionais, em especial na Mata Atlântica, possuindo elevada representatividade entre os elementos do estrato arbóreo tanto nas formações costeiras quanto nas semidecíduas do estado de Minas Gerais (LIMA, 2000; OLIVEIRA-FILHO; FONTES, 2000; LOMBARDI; GONÇALVES, 2000; MEIRANETO; MARTINS, 2002; LOPES et al., 2002; MARANGON et al., 2003; SOARES et al., 2006; FERREIRA JUNIOR et al., 2007).

O monofiletismo de Fabaceae (Leguminosae) e das subfamílias Mimosoideae e Papilionoideae e o parafiletismo de Caesalpinioideae, são reconhecidos pela maioria dos autores que se baseiam em dados macromoleculares e em cladística (DOYLE et al., 2000; WOJCIECHOWSKI, 2003; WOJCIECHOWSKI et al., 2004; LEWIS et al., 2005; SOUZA; LORENZI, 2008).

Mimosoideae com 78 gêneros e aproximadamente 3.270 espécies (LEWIS et al., 2005), estão distribuídas nas regiões tropicais, subtropicais e cálido-temperadas (POLHILL; RAVEN, 1981) e cerca de dois terços das espécies conhecidas estão subordinadas a três gêneros: Senegalia, Mimosa e Inga (ELIAS, 1974).

Trabalhos mais recentes, envolvendo estudos taxonômicos sobre a família Fabaceae e similaridade florística em remanescentes de Floresta Estacional Semidecídua, próximo ao local do presente estudo incluem os de Bortoluzzi (2004), Nunes (2003) e Bosquetti (2004). Bortoluzzi (2004) encontrou para o Parque Estadual do Rio Doce (PERD) 40 espécies de Papilionoideae reunidas em 21 gêneros, já Nunes (2003), estudando as Mimosoideae do PERD, encontrou 34 táxons reunidos em 33 espécies e 14 gêneros, enquanto Bosquetti (2004) encontrou 28 espécies de Caesalpinioideae reunidas em 15 gêneros.

Considerando a importância das florestas nativas, no cenário estratégico, ambiental, social e econômico do país e as restrições ambientais sobre o uso das florestas nativas, estudos da composição e similaridade florística de um ambiente permitem o mapeamento da área e fornecem subsídios para elaboração de planos de manejo florestal para utilização sustentável do recurso. A análise das relações de similaridade florística entre comunidades geralmente conduz ao estabelecimento de padrões, condicionados por fatores diversos que determinam a ocorrência ou não das espécies em diferentes locais (DURIGAN et al., 2008).

O estudo da diversidade florística na região do Manso, além de contribuir com o conhecimento sobre as Fabaceae (Leguminosae), especificamente da flora de Minas Gerais, é importante para o conhecimento dessa família nos remanescentes de Floresta Estacional Semidecídua das regiões montanas do estado, especialmente na região do Vale do Aço.

O presente estudo tem como objetivos verificar a distribuição geográfica das espécies de Mimosoideae encontradas na região do Manso, no município de Antônio Dias e comparar a similaridade na composição florística desta subfamília entre a região do Manso e outras áreas de floresta atlântica do Sudeste do Brasil.

\section{MATERIAL E MÉTODOS}

Área de estudo

A área de estudo está localizada na região do Manso (19 $\left.41^{\prime} 15^{\prime} \mathrm{S}, 42^{\circ} 48^{\prime} 45^{\prime \prime} \mathrm{W}\right)$, área rural 
do município de Antônio Dias, leste de Minas Gerais, com altitudes variando entre 625 e 785 metros. Sua vegetação está situada no domínio da floresta atlântica e pode ser classificada, de acordo com Veloso et al. (1991), como floresta estacional semidecídua montana.

O relevo da área representa parte dos Planaltos Dissecados do Centro Sul e do Leste de Minas Gerais, coberto originalmente pela Mata Atlântica e substituído por pastagens e por monoculturas em função das atividades econômicas que se sustentaram desde a colonização até os dias atuais. Assim, seus fragmentos florestais apresentam-se hoje isolados em capões nos altos dos morros, em algumas encostas mais íngremes e em trechos descontínuos ao longo dos rios (FUNDAÇÃO CENTRO TECNOLÓGICO DE MINAS GERAIS, 1982).

O clima, segundo o sistema de Köppen (1948), é do tipo Aw, caracterizando um clima tropical úmido de savana, megatérmico. O regime pluviométrico apresenta variação de 1000 a 1200 $\mathrm{mm}$ de precipitação anual e deficiência hídrica da ordem de 50 a $100 \mathrm{~mm}$, assim como, excedentes hídricos de 100 a 200 mm (EMATER, 1980).

\section{Coleta de dados}

Os dados de ocorrência dos 9 táxons de Mimosoideae encontrados na região do Manso, Antônio Dias - MG foram obtidos através do estudo florístico e taxonômico da subfamília, realizado por Silva (2006). As coletas de material botânico foram realizadas por trilhas e caminhos, sem prévia delimitação de área, mensalmente entre os meses março de 2005 e fevereiro de 2006 em 8 trilhas, e sempre que possível, adentrando cerca de $20 \mathrm{~m}$ em ambos os lados delas, com o intuito de ampliar a área de coleta. Coletas esporádicas também foram realizadas em outras trilhas presentes nessa região.

A classificação genérica está de acordo com Lewis et al. (2005) e os nomes das espécies foram atualizados consultando revisões taxonômicas e o Ildis (2006), além de consultas aos Herbários do Centro Universitário do Leste de Minas Gerais e da Universidade Federal de Viçosa.

Os dados referentes à distribuição geográfica dos táxons foram obtidos de revisões taxonômicas e de levantamentos florísticos. Cinco padrões de distribuição foram reconhecidos segundo a faixa predominante de ocorrência, na qual os táxons exibem distribuições contínuas ou não e adaptados de Lima e Guedes-Bruni (1997); Lima (2000); Morim (2006) e Nunes et al. (2007). Foram observados os seguintes padrões: 1. Anfiatlântico; 2. Neotropical; 3. América do Sul OcidentalCentro-Oriental; 4. Brasil Centro-Oriental; 5. Brasil Atlântico Nordeste-Sudeste.

Quanto à preferência por habitat, as espécies foram classificadas de acordo com os diferentes domínios vegetacionais brasileiros definidos por Joly et al. (1999), em elementos florísticos generalistas, aquelas que além do Domínio Atlântico ocorrem em outros Domínios como Amazônico, Cerrado e/ ou Caatinga ou especialistas, espécies exclusivas do Domínio Atlântico.

A similaridade na composição de espécies arbóreas de Mimosoideae foi realizada entre a região do Manso e as seguintes localidades: Parque Estadual do Rio Doce (PERD) - MG; RPPN Feliciano Miguel Abdala, conhecida como Reserva Biológica de Caratinga (RBC) - MG; Floresta do Jardim Botânico da Universidade Federal de Viçosa (UFV) - MG; Reserva Florestal da ESAL, Lavras (ESAL) - MG; Serra de São José, Tiradentes (SSJ) - MG; Mata do Galego, Luminária (L/MG) - MG; Herbário da Reserva Florestal de Linhares, Companhia Vale do Rio Doce (CVRD) - ES; São Carlos (SC/SP) - SP; Pedreira (P/SP) - SP; Parque Estadual das Fontes do Ipiranga (PEFI) - SP; Macaé de Cima (MC/RJ) - RJ. As fitofisionomias dessas áreas foram identificadas de acordo com Veloso et al. (1991).

A matriz simétrica com os índices de similaridade de Sorensen foi calculada através da informação florística, organizada em nível de espécie, em uma matriz de presença e ausência. Para que os agrupamentos pudessem ser visualizados foi construído o dendrograma (Figura 1) a partir do método de algoritmo de médias não ponderadas (UPGMA) (SNEATH; SOKAL, 1973).

\section{RESULTADOS E DISCUSSÃO}

\section{Padrões de distribuição geográfica}

A amplitude de distribuição geográfica dos táxons de Mimosoideae da região do Manso, Antônio Dias, abrange 3 macrorregiões (Tabela 1): Américas e África (22,22\%); limitados à América do Sul $(44,45 \%)$ e restritos ao Brasil $(33,33 \%)$.

Observou-se que $100 \%$ do total de espécies estudadas são generalistas (Tabela 2). Estudos realizados por Morim (2006) nas formações 
TABELA 1: Número e porcentagem dos táxons de Mimosoideae da região do Manso, Antônio Dias, distribuídas por macrorregiões.

TABLE 1: Number and percentage of Mimosoideae taxa of Manso's region, Antonio Dias, distributed for macroregions.

\begin{tabular}{lcc}
\hline \multicolumn{1}{c}{ Macrorregiões } & Número de táxons & Porcentagem (\%) \\
\hline Américas e África & 2 & 22,22 \\
Limitados a América do Sul & 4 & 44,45 \\
Restritos do Brasil & 3 & 33,33 \\
\hline \multicolumn{1}{c}{ Total } & 9 & 100 \\
\hline
\end{tabular}

ombrófilas montanas e alto-montanas do Parque Nacional do Itatiaia (RJ) e por Nunes et al. (2007) na floresta semidecídua submontana do Parque Estadual do Rio Doce (PERD/MG), encontraram espécies especialistas de formações domínio Atlântico, além das generalistas, embora o maior número delas estivesse também concentrado neste último tipo, demonstrando a dominância de espécies generalistas nos habitat.

O padrão Anfiatlântico (Tabela 2) abrange espécies com faixa de ocorrência nas Américas e África, representando $11,11 \%$ das Mimosoideae encontradas no Manso. Faz parte desse padrão o táxon: Mimosa pellita var. pellita. No Manso, essa espécie foi encontrada na borda de fragmentos, no qual a pressão antrópica se encontra mais intensa.

TABELA 2: Padrões de distribuição, relação dos táxons quanto à preferência de habitat das Mimosoideae do Manso.

TABLE 2: Standards of distribution, relation of taxa how much the habitat preference of Manso's Mimosoideae.

\begin{tabular}{|c|c|c|c|}
\hline \multirow{2}{*}{$\begin{array}{l}\text { Padrões de distribuição } \\
\qquad\left(\mathrm{n}^{\mathrm{o}} \text { de espécies }\right)\end{array}$} & \multirow{2}{*}{ Táxons Referências } & \multicolumn{2}{|c|}{ Elementos Florísticos } \\
\hline & & Especialistas & Generalistas \\
\hline Anfiatlântico (1) & $\begin{array}{l}\text { Mimosa pellita Humb. \& Bonpl. ex Willd. } \\
\text { var. pellita }{ }^{3 ; 4 ; 6 ; ; ; 11}\end{array}$ & & $\mathrm{X}$ \\
\hline \multirow{2}{*}{ Neotropical (2) } & Anadenanthera peregrina (L.) Speg. ${ }^{1 ; 10 ; 15 ; 16}$ & & $\mathrm{X}$ \\
\hline & Inga vera subsp. affinis (DC.) T.D. Penn. ${ }^{14 ; 18}$ & & \\
\hline \multirow{4}{*}{$\begin{array}{l}\text { América do Sul Ocidental- } \\
\text { Centro-Oriental (3) }\end{array}$} & Anadenanthera colubrina (Vell.) Brenan var. & & $\mathrm{X}$ \\
\hline & cebil (Griseb.) Altschul 1;17 & & $\mathrm{X}$ \\
\hline & $\begin{array}{l}\text { Mimosa debilis Humb. \& Bonpl. ex Willd. } \\
\text { Var. cebil } 7 ; 5\end{array}$ & & $\mathrm{X}$ \\
\hline & $\begin{array}{l}\text { Piptadenia gonoacantha (Mart.) J.F. Macbr., } \\
\text { Contrib. }{ }^{2 ; 5 ; 8 ; 13 ; 19}\end{array}$ & & $\mathrm{X}$ \\
\hline \multirow[t]{2}{*}{ Brasil Centro-Oriental (2) } & $\begin{array}{l}\text { Senegalia martiusiana (Steud.) Seigler \& } \\
\text { Ebingler } 5 ; 8 ; 13\end{array}$ & & $\mathrm{X}$ \\
\hline & Plathymenia reticulata Benth., in Hook. ${ }^{5 ; 10 ; 13}$ & & $\mathrm{X}$ \\
\hline $\begin{array}{l}\text { Brasil Atlântico Nordeste- } \\
\text { Sudeste (1) }\end{array}$ & Stryphnodendron polyphyllum Mart. ${ }^{10 ; 12 ; 13}$ & & $\mathrm{X}$ \\
\hline
\end{tabular}

Em que: ${ }^{1}$ Altschul (1964); ${ }^{2}$ Barroso (1965); ${ }^{3}$ Bässler (1985); ${ }^{4}$ Barneby (1991); ${ }^{5}$ Bentham (1875); ${ }^{6}$ Brenan (1955);

${ }^{7}$ Burkart (1948); ${ }^{8}$ Burkart (1979); Isely (1998); ${ }^{10}$ Lewis (1987); ${ }^{11}$ Leogier (1988); ${ }^{12}$ Martins (1981); ${ }^{13}$ Mendonça Filho (1996); ${ }^{14}$ Pennington (1997); ${ }^{15}$ Oliveira-Filho e Fontes (2000); ${ }^{16}$ Nunes et al. (2007); ${ }^{17}$ Prado e Gibbs (1993); ${ }^{18}$ Garcia (1998); ${ }^{19}$ Lorenzi (1992). 
Pode ser encontrada no Caribe (LEOGIER, 1988) e EUA(ISELY, 1998). Encontra-se também distribuída pelo México e América Central entre as encostas do Pacífico e Atlântico; para o sul de Sinaboa e sul de Veracruz, sudeste do Panamá e em terrenos baldios de Cuba (BÄSSLER, 1985). Na América do Sul e em terras baixas do Pacífico para noroeste da Colômbia mais ou menos a $8^{\circ} \mathrm{S}$ e noroeste do Peru, interior leste da Colômbia e Venezuela até as Guianas (BARNEBY, 1991). Está dispersa ao longo dos principais rios da Amazônia brasileira, ao longo da costa do Rio de Janeiro, estendendo ao sul da linha tropical da costa de Santa Catarina, oeste do Paraná e Paraguai, do sul ao leste da Argentina (Formosa, Chaco, Corrientes, Misiones, Entre Rios), atingindo $35^{\circ} \mathrm{S}$ do delta do Rio Paraná (BARNEBY, 1991). No velho mundo ela é encontrada também na África tropical, Madagascar e introduzida em Java (BRENAN, 1955).

Opadrão Neotropical (Tabela 2) compreende espécies com faixa de distribuição nos trópicos da América do Sul, podendo se estender até América Central. Inclui-se nesse padrão Anadenanthera peregrina e Inga vera subsp. affinis, que representa $22,22 \%$ dos táxons encontrados. No Manso Anadenanthera peregrina foi encontrada apenas na borda da estrada de acesso à Fazenda. Nunes et al. (2007) registraram sua ocorrência não só às margens de estradas que cortam o PERD como também no interior da floresta. Pode ser encontrada nas Ilhas do Caribe e em países da América do Sul, sendo estes: Brasil, Colômbia, Guiana Francesa, Guiana, Paraguai, Suriname e Venezuela (ALTSCHUL, 1964; LEWIS, 1987). Oliveira-Filho e Fontes (2000), registraram sua ocorrência tanto em florestas semidecíduas montanas como submontanas.

No Manso, a espécie Inga vera subsp. affinis foi encontrada em todas as trilhas percorridas, tanto na borda do fragmento, quanto no interior deste e em locais de maior altitude (775 m). Ocorre na América do Sul, em toda a região tropical, com exceção das regiões áridas do Nordeste e Planalto Central do Brasil que se estende até o Chaco na Argentina (PENNINGTON, 1997). No Brasil, suas populações ocupam tanto ambientes higrófilos como chegam também aos mais áridos como caatinga e cerrados, nos quais estão sempre associados à margem de rios perenes ou temporários (GARCIA, 1998).

O padrão América do Sul Ocidental-CentroOriental (Tabela 2) inclui táxons cuja distribuição alcança áreas do oeste, centro e leste da América do Sul. O extremo mais ao sul é a Argentina que também é atingida por alguns táxons. São reconhecidos nesse padrão 3 táxons (33,33\%): Anadenanthera colubrina var. cebil, Mimosa debilis var. debilis e Piptadenia gonoacantha.

Anadenanthera colubrina var. cebil no Manso foi encontrada na estrada de acesso à Fazenda Sertão do Manso. Ocorrente na Argentina, Bolívia, Brasil (Bahia, Ceará, Maranhão, Mato Grosso, Minas Gerais, Pernambuco, Piauí, Rio de Janeiro e São Paulo), Paraguai e Peru (ALTSCHUL, 1964). Tem como provável local de origem as terras altas da região Sudeste do Brasil que são consideradas também o centro de origem do gênero (ALTSCHUL, 1964).

Mimosa debilis var. debilis, na região do Manso, é encontrada na estrada de acesso à Fazenda Sertão do Manso e está dispersa apenas nas bordas do fragmento, sugerindo ser uma espécie pioneira. Pode ser encontrada na Argentina, Bolívia, Brasil e Paraguai (BURKART, 1948). No Brasil ela é encontrada em Minas Gerais e São Paulo (BENTHAM, 1875).

No Manso, Piptadenia gonoacantha tem ampla distribuição, sendo encontrada em todas as trilhas percorridas, mas sua frequência é maior nas bordas do fragmento. Na América Latina ocorre nos seguintes países: Bolívia, Brasil e Paraguai (BURKAT, 1979). No Brasil ela ocorre nos estados do Rio de Janeiro, São Paulo, Bahia, Piauí, Pernambuco e Minas Gerais (BENTHAM, 1875; BARROSO, 1965; MENDONÇA FILHO, 1996), em florestas de galeria no cerrado e floresta pluvial Atlântica (LORENZI, 1992; MENDONÇA FILHO et al., 1998). Nunes et al. (2007), também registraram sua ocorrência no interior da floresta em ambientes úmidos e secos.

No padrão Brasil Centro-Oriental (Tabela 2) são inseridas espécies com distribuição nas regiões centro-oeste, nordeste, sudeste e/ou sul do Brasil. Padrão observado em 2 espécies (22,22\%): Senegalia martiusiana e Plathymenia reticulata. $\mathrm{Na}$ região do Manso, Senegalia martiusiana encontrase próxima à porteira da Fazenda Sertão do Manso, sendo de localização restrita. Ocorre no Paraná, Santa Catarina, Rio de Janeiro, Minas Gerais e Goiás (BENTHAM, 1875; BURKART, 1979; MENDONÇA FILHO, 1996).

Plathymenia reticulata, na região do Manso, encontra-se na estrada de acesso à Fazenda Sertão do Manso. Pode ser encontrada nos estados da Bahia, Piauí, Ceará, Goiás e Minas Gerais (BENTHAM, 1875; LEWIS, 1987; MENDONÇA 
FILHO, 1996), ou seja, o sudeste é seu limite de ocorrência, não estendendo sua distribuição até a região sul do Brasil.

O padrão Brasil Atlântico Nordeste-Sudeste (Tabela 2) abrange a faixa de distribuição desde o nordeste ao sudeste brasileiro. Foi verificada apenas uma espécie $(11,11 \%)$, Stryphnodendron polyphyllum. No Manso, tem uma ampla distribuição, sendo encontrada desde as bordas de estradas a cerca de $625 \mathrm{~m}$ de altitude até as regiões mais altas $(785 \mathrm{~m})$, menos antropizadas. Ocorre nos estados de Pernambuco, Bahia, Minas Gerais e Rio de Janeiro (MARTINS, 1981; LEWIS, 1987; MENDONÇA FILHO, 1996).

\section{Similaridades florísticas}

A análise de agrupamento das áreas estudadas com base nas espécies de Mimosoideae (Figura 1) revelou dois grandes blocos em nível de 0,21 da escala de Sorensen. O primeiro deles, aqui denominado bloco $\mathrm{A}$, reunindo as áreas do Manso, SSJ, ESAL, UFV, L/MG, P/SP, SC/SP, PEFI, MC/ $\mathrm{RJ}$ e o segundo, bloco B reunindo o PERD, RBC e CVRD.

As formações vegetacionais dos estados de Minas Gerais (Manso, SSJ, ESAL, UFV, L/MG), São Paulo (P/SP, SC/SP, PEFI) e Rio de Janeiro (MC/ $\mathrm{RJ})$ que são: floresta ombrófila densa alto-montana e montana e a floresta estacional semidecídua montana, foram reunidas no bloco A. Enquanto o bloco B reuniu aquelas dos estados de Minas Gerais (PERD, RBC) e do Espírito Santo (CVRD), que são: floresta estacional semidecídua submontana e floresta ombrófila densa das terras baixas.

A região do Manso e a Serra de São José, Tiradentes, MG (SSJ), do bloco A, foram as áreas que apresentaram a flora mais semelhante formando um grupo em nível de 0,71 , ambos com tipologia de floresta estacional semidecídua montana (VELOSO et al., 1991). A presença de mesma tipologia florestal e faixas altitudinais elevadas (Tabela 3) justificam a semelhança florística entre as duas áreas. Apresentaram em comum os seguintes táxons: Anadenanthera colubrina, Anadenanthera peregrina, Inga vera subsp. affins, Piptadenia gonoacantha, Plathymenia reticulata e Stryphnodendron polyphyllum.

Esse grupo se juntará a ESAL, em nível de 0,59 , que está localizada no sul do estado de Minas Gerais, apresentando a mesma tipologia do grupo. Os táxons comuns entre essas três áreas foram:
Anadenanthera peregrina, Inga vera subsp. affinis e Piptadenia gonoacantha.

O terceiro grupo reuniu a área da Reserva Florestal da ESAL, SSJ e Manso com a área denominada Jardim Botânico da UFV, localizado na zona da Mata Mineira e tipologia de floresta estacional semidecídua submontana (VELOSO et al., 1991). Esse grupo foi isolado, em nível de 0,41 e apresentou em comum os seguintes táxons: Inga vera subsp. affinis e Piptadenia gonoacantha, sendo que nenhuma delas de ocorrência restrita para essas localidades. A baixa similaridade encontrada entre esse grupo, parecem ter sido influenciada pela diferença altitudinal entre as áreas principalmente em relação ao Manso. A importância da altitude na diferenciação florística de florestas estacionais foi relatada para estas formações na Região Sudeste do Brasil (OLIVEIRA-FILHO et al., 1994).

O quarto grupo reuniu a área da Mata do Galego, da região da Bacia do Rio Ingaí, com aquela localizada no município de Pedreira em São Paulo, ambas com tipologia de floresta estacional semidecídua montana (VELOSO et al., 1991) e a UFV, ESAL, SSJ e Manso. Esse grupo foi isolado em nível de 0,39 , ou seja, com baixa similaridade. A diferença entre a metodologia empregada nos inventários analisados nesta comparação (Tabela 3) pode estar influenciando a baixa similaridade encontrada.

O quinto grupo liga o município de São Carlos, em São Paulo, com tipologia de Floresta Estacional Semidecídua Montana (VELOSO et al., 1991) aos grupos 1, 2, 3 e 4. Todos estão em regiões com faixas altitudinais elevadas (entre 700 e $1001 \mathrm{~m} / \mathrm{alt}$.), mas esse grupo foi isolado, em nível de 0,29 . As espécies responsáveis pela similaridade entre essas áreas foram: Inga sessilis e Piptadenia gonoacantha.

$\mathrm{O}$ sexto grupo ligou a área do Parque Estadual das Fontes do Ipiranga (PEFI) com tipologia de floresta estacional semidecídua montana, e a área denominada Reserva Ecológica de Macaé de Cima, localizada no distrito de Macaé de Cima, Rio de Janeiro, com tipologia de floresta ombrófila densa montana. Esse grupo foi isolado, em nível de 0,41. A diferença entre o esforço de coleta e a metodologia empregada (Tabela 3) pode estar influenciando a baixa similaridade, embora ambos tenham enfocado apenas as Fabaceae (Leguminosae) das referidas regiões; fato também observado por Nunes et al. (2007). As espécies responsáveis pela similaridade entre essas áreas foram: Abarema langsdorfii, Inga 
TABELA 3: Quadro comparativo do número de espécies arbóreas em levantamentos florísticos de Mimosoideae, em diversas áreas.

TABLE 3: Comparative picture of the number of arboreal species in floristic surveys of Mimosoideae, in several areas.

\begin{tabular}{|c|c|c|c|c|c|}
\hline Autores & Localidade & Espécies & Alt. (m) & Amostragem & Sigla \\
\hline 1. Silva $(2006)^{1}$ & Região do Manso, Antônio Dias - MG. & 09 & $625-785$ & IV & Manso \\
\hline 2. Nunes et al. (2007) & Parque Estadual do Rio Doce, PERD - MG. & 25 & $250-380$ & IV & PERD \\
\hline $\begin{array}{l}\text { 3. Mendonça Filho } \\
\text { (1996) }\end{array}$ & $\begin{array}{l}\text { RPPN Feliciano Miguel Abdala, Caratinga } \\
\text { - MG. }\end{array}$ & 20 & $318-628$ & I & $\mathrm{RBC}$ \\
\hline $\begin{array}{l}\text { 4. Sevilha et al. } \\
\text { (2001) }\end{array}$ & $\begin{array}{c}\text { Floresta do Jardim Botânico da UFV, } \\
\text { Viçosa - MG. }\end{array}$ & 09 & 700 & III & UFV \\
\hline $\begin{array}{l}\text { 5. Oliveira-Filho et } \\
\text { al. (1994) }\end{array}$ & Reserva Florestal da ESAL, Lavras - MG. & 06 & 925 & III & ESAL \\
\hline $\begin{array}{l}\text { 6. Oliveira-Filho e } \\
\text { Machado (1993) }\end{array}$ & Serra de São José, Tiradentes - MG. & 10 & 900 & III & SSJ \\
\hline $\begin{array}{l}\text { 7. Rodrigues et al. } \\
\text { (2003) }\end{array}$ & Mata do Galego, Luminárias - MG. & 10 & $\begin{array}{l}880- \\
1001\end{array}$ & III & $\mathrm{L} / \mathrm{MG}$ \\
\hline $\begin{array}{l}\text { 8. Jesus e Garcia } \\
(1992)\end{array}$ & $\begin{array}{l}\text { CVRD-Herbário da Reserva Florestal de } \\
\text { Linhares - ES. }\end{array}$ & 24 & $28-65$ & II & CVRD \\
\hline $\begin{array}{l}\text { 9. Silva e Soares } \\
(2003)\end{array}$ & São Carlos - SP. & 06 & 850 & III & $\mathrm{SC} / \mathrm{SP}$ \\
\hline $\begin{array}{l}\text { 10. Yamamoto et al. } \\
\text { (2005) }\end{array}$ & Pedreira - SP. & 07 & $750-850$ & III & $\mathrm{P} / \mathrm{SP}$ \\
\hline $\begin{array}{l}\text { 11. Custódio Filho e } \\
\text { Mantovani (1986) }\end{array}$ & $\begin{array}{l}\text { Parque Estadual das Fontes do Ipiranga - } \\
\text { SP. }\end{array}$ & 09 & 798 & I & PEFI \\
\hline $\begin{array}{l}\text { 12. Lima e Guedes- } \\
\text { Bruni (1997) }\end{array}$ & Macaé de Cima - RJ & 14 & $\begin{array}{l}880- \\
1720\end{array}$ & III & $\mathrm{MC} / \mathrm{RJ}$ \\
\hline
\end{tabular}

${ }^{1}$ Presente Trabalho; 1-3, 8 e 11.Trabalhos específicos com Leguminosae.

Em que: Alt.: altitude em metros; Tipo de amostragem realizada: I: Inventário e estudo taxonômico de Leguminosae; II: Inventário de Leguminosae a partir de levantamento de herbário; III: Inventário florístico e Fitossociológico; IV: Inventário e estudo taxonômico de Mimosoideae (Leguminosae).

capitata, Inga sessilis e Piptadenia gonoacantha. Somente Piptadenia gonoacantha foi compartilhada com o Manso.

A baixa similaridade entre o PEFI e o Manso, 0,40 da escala de Sorensen, ambos como o mesmo tipo de formação florestal pode estar relacionada à maior distância do oceano em que encontra o Manso. Segundo Oliveira-Filho e Fontes (2000), esse fator corresponde ao aumento de duração da estação seca, podendo ser responsável pelas diferenças entre as floras de florestas semidecíduas. Também houve baixa similaridade entre o Manso e Macaé de Cima, 0,19 da escala de Sorensen. O principal fator que parece ter influenciado esse resultado foi a elevada altitude encontrada em Macaé de Cima (Tabela 3).
O Parque Estadual do Rio Doce (PERD) e a RPPN Feliciano Miguel Abdala (RBC), foram as áreas que apresentaram a flora mais semelhante formando um grupo em nível de 0,80. Estas áreas estão localizadas na região leste de Minas Gerais e possuem mesma tipologia florestal, ou seja, estacional semidecídua submontana (VELOSO et al., 1991). A presença de mesma tipologia florestal, a proximidade entre as áreas e a localização na mesma bacia hidrográfica justificam esta semelhança, que também foi detectada por Nunes et al. (2007). Apenas Abarema obovata tem ocorrência exclusiva para estas duas áreas. As espécies Anadenanthera colubrina, Anadenanthera peregrina, Piptadenia gonoacantha, Plathymenia 


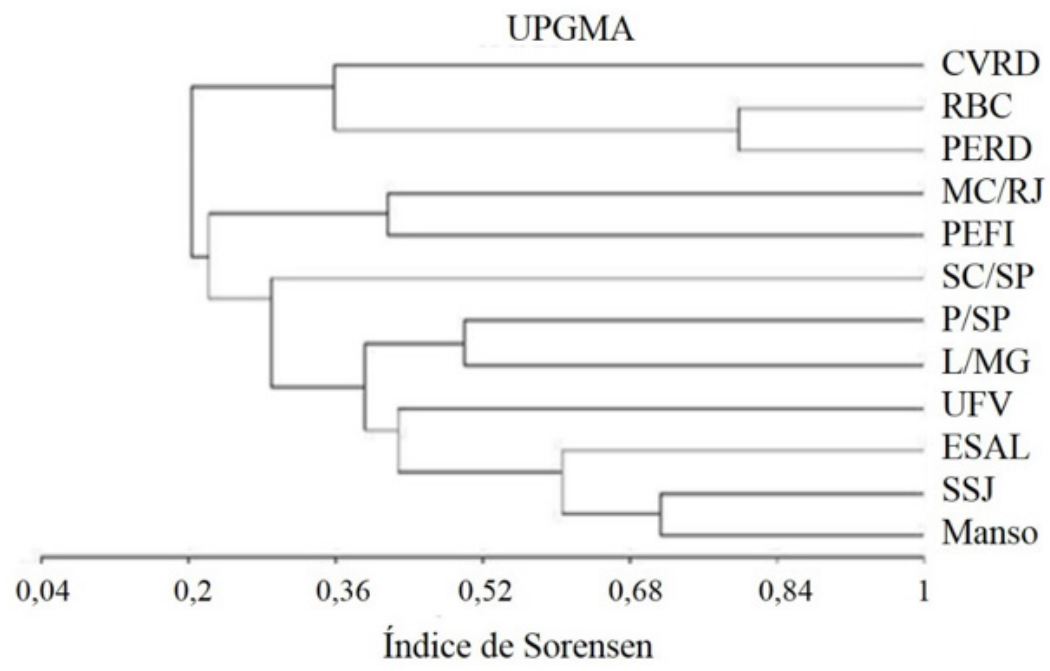

FIGURA 1: Dendrograma apresentado similaridade florística entre as regiões de Floresta Atlântica dos estados do Espírito Santo, Minas Gerais, Rio de Janeiro e São Paulo, utilizando como coeficiente o índice de Sorensen e agrupando as localidades pelo método de média de grupo (UPGMA). CVRD - Herbário da Reserva Florestal de Linhares - ES; RBC - RPPN Feliciano Miguel Abdala, Caratinga - MG; PERD - Parque Estadual do Rio Doce - MG; MC/RJ - Macaé de Cima - RJ; PEFI - Parque Estadual das Fontes do Ipiranga - SP; SC/SP - São Carlos - SP; P/SP - Pedreira - SP; L/MG - Mata do Galego, Luminárias - MG; UFV - Floresta do Jardim Botânico da UFV, Viçosa - MG; ESAL - Reserva Florestal da ESAL, Lavras - MG; SSJ - Serra de São José, Tiradentes - MG; Manso - Região do Manso, Antônio Dias - MG.

FIGURE 1: Dendrogram presenting floristic similarity enters the regions of Atlantic Forest of the states of Espírito Santo, Minas Gerais, Rio de Janeiro and São Paulo, using as coefficient the index of Sorensen and grouping the localities for the method of group average (UPGMA). CVRD - Herbarium of the Linhares Forest Reserve, ES; RBC - RPPN Feliciano Miguel Abdala, Caratinga, MG; PERD - Rio Doce State Park, MG; MC/RJ - Macaé de Cima, RJ; PEFI Fontes do Ipiranga State Park, SP; SC/SP - São Carlos, SP; P/SP - Pedreira, SP; L/MG - Mata do Galego, Luminárias, MG; UFV - Botanical Garden of UFV, Viçosa, MG; ESAL - ESAL Forest Reserve, Lavras, MG state; SSJ - Serra de São José, Tiradentes, MG state; Manso Region of Manso, Antônio Dias, MG state.

reticulata, e Stryphnodendron polyphyllum também foram encontradas no Manso, além dos dois locais. Comparando a composição florística do PERD e $\mathrm{RBC}$ em relação à região do Manso e levando-se em consideração que estão próximas geograficamente e localizadas dentro da mesma bacia hidrográfica, foi esperado que apresentassem um índice de semelhança elevado. Ao contrário, pôde-se observar que o índice de ligação foi baixo entre elas, 0,38 , provavelmente em decorrência das duas primeiras estarem localizadas em regiões mais baixas em relação à última. A importância da altitude na diferenciação florística de florestas estacionais foi relatada por Oliveira-Filho et al. (1994).

Esse grupo se juntará a CVRD, em nível de 0,36 , que está localizada na cidade de Linhares, norte do Espírito Santo, na porção baixa da bacia do Rio Doce, com vegetação de floresta ombrófila densa de terras baixas. As espécies comuns entre essas três áreas foram: Senegalia polyphylla, Albizia polycephala, Inga capitata, Inga edulis, Inga flagelliformis, Inga leptantha, Inga thibaudiana, Parapiptadenia pterosperma e Pseudopiptadenia contorta. Dessas, três são de ocorrência restrita para essas localidades: Inga edulis, Inga flagelliformis e Inga thibaudiana, que ocorrem tanto em florestas ombrófilas quanto em florestas estacionais (GARCIA, 1998). Dentre os táxons compartilhados entre o PERD e CVRD, somente Zygia latifolia var. glabrata, táxon restrito às florestas ripárias (BARNEBY; GRIMES, 1996) foi de ocorrência exclusiva para ambas as localidades. 
Não houve nenhuma similaridade florística entre o Manso e a CVRD, em nível de 0,00. A diferença altitudinal (Tabela 3) e de formação florestal entre essas áreas parecem ser os principais fatores que influenciaram a falta de ligação entre elas.

Observando o dendrograma (Figura 1) e analisando os grupos, pode-se verificar que as formações florestais localizadas nas regiões mais baixas isolaram-se daquelas localizadas nas regiões mais altas. A presença de um período de baixas temperaturas nas florestas montanas pode estar diferenciando as duas floras ocorrentes nessas formações. Fato também observado por OliveiraFilho et al. (1994) quando analisaram 30 áreas florestais da costa em direção ao interior do Brasil.

Segundo Nunes et al. (2007), a presença de algumas espécies, somente nas áreas com altitudes elevadas, em florestas de planícies e pequenas elevações costeiras ou em baixas altitudes como vales de bacias hidrográficas provavelmente estão relacionadas à preferência de habitat de algumas espécies determinando uma singularidade florística para essas áreas em função das restrições climáticas e edáficas a que estão submetidas.

\section{CONCLUSÃO}

O padrão predominante de distribuição geográfica dos nove táxons foi: América do Sul Ocidental-Centro-Oriental $(33,33 \%)$, sendo este também o padrão predominante dos táxons generalistas e não houve ocorrência de táxons especialistas em nenhum dos padrões.

Existe uma indicação de ligação florística entre a floresta estacional semidecídua do Manso e as outras formações de Floresta Atlântica analisadas, com exceção da floresta ombrófila densa de terras baixas, corroborando com a proposição de OliveiraFilho e Fontes (2000) de que a flora arbórea da floresta semidecídua seja um subconjunto da flora das florestas ombrófilas.

\section{AGRADECIMENTOS}

Os autores agradecem à Fundação Geraldo Perlingeiro de Abreu/Unileste - MG pela concessão da bolsa de iniciação científica para a primeira autora e ao Sr. Carlos, proprietário da Fazenda Sertão do Manso, por autorizar a execução do trabalho em sua propriedade.

\section{REFERÊNCIAS BIBLIOGRÁFICAS}

ALTSCHUL, S. V. R. A taxonomic study of the genus Anadenanthera. Contributions from the Gray Herbarium of Harvard University, Cambridge, v. 193, p. 1-65, 1964.

BARNEBY, R. C. Sensitivae censitae: a description of the genus Mimosa Linnaeus (Mimosaceae). New World. Memoirs of the New York Botanical Garden, New York, v. 65, p. 835, 1991.

BARNEBY, R. C.; GRIMES, W. Silk tree, guanacaste, monkey's earring: a generic system for the synandrous Mimosaceae of the Americas. Part I. Abarema, Albizia, and Allies. Memoirs of the New York Botanical Garden, New York, v. 74, n. 1, p. 1-292, 1996.

BARROSO, G. M. Leguminosas da Guanabara. Arquivos do Jardim Botânico do Rio de Janeiro, Rio de Janeiro, v. 18, p. 115-118, 1965.

BÄSSLER, M. Die Gattung Mimosa (Legminosae - Mimosoideae) in Cuba. Repertorium Specierum Novarum Regni Vegetabilis, Beihefte, v. 96, p. 581-611, 1985.

BENTHAM, G. Leguminosae Mimosoideae. In: MARTIUS, C. Flora Brasiliensis, Monachii, v. 5, n. 2, p. 458-500, 1875.

BORTOLUZZI, R. L. C. Leguminosae, Papilionoideae no Parque Estadual do Rio Doce, Minas Gerais, Brasil. II: árvores e arbustos escandentes. Acta Botânica Brasílica, Porto Alegre, v. 18, n. 1, p. 49-71, 2004.

BOSQUETTI, L. B. Caesalpinioideae (Leguminosae) no Parque Estadual do Rio Doce, Minas Gerais, Brasil. 2004. 79 f. Dissertação (Mestrado em Botânica) - Universidade Federal de Viçosa, Viçosa, 2004.

BRENAN, J. P. M. Mimosa. Notes on Mimosoideae 1, Kew Bull, p. 184-189, 1955.

BURKART, A. Las leguminosas argentinas. Buenos Aires: ACME Agency, 1948. 569 p.

BURKART, A. Leguminosas, Mimosoidea. In: Flora Ilustrada Catarinense. Florianópolis: Herbário Barbosa Rodrigues, 1979. 299 p.

FUNDAÇÃO CENTRO TECNOLÓGICO DE MINAS GERAIS. Diagnóstico Ambiental de Minas Gerais. Belo Horizonte: CETEC, 1982. CUSTÓDIO FILHO, A.; MANTOVANI, W. Flora Fanerogâmica da Reserva do Parque Estadual das Fontes do Ipiranga (São Paulo, Brasil). 81-Leguminosae. Hoehnea, São Paulo, v. 13, p. 113-140, 1986.

DOYLE, J. J. et al. Towards a comprehensive 
phylogeny oflegumes: evidence from $r b c L$ sequences and non-molecular data. In: HERENDEEN, P. S.; BRUNEAU, A. (Eds.). Advances in Legume Systematics. Kew: Royal Botanic Gardens, 2000. p. 151-163.

DURIGAN, G. et al. Estádio sucessional e fatores geográficos como determinantes da florística entre comunidades florestais no Planalto Atlântico, Estado de São Paulo, Brasil. Acta Botânica Brasílica, Porto Alegre, v. 22, n. 1, p. 51-62, 2008.

ELIAS, T. S. The genera of Mimosoideae (Leguminosae) in the southeastern United States. Journal Arnold Arboretum, Cambridge, v. 55, n. 1, p. 67-118, 1974.

EMATER. Zoneamento Agroclimático do Estado de Minas Gerais. Belo Horizonte: Secretaria de Estado da Agricultura, MG, 1980. 114 p.

FERREIRA JÚNIOR, W. G. et al. Composição florística da vegetação arbórea de um trecho de floresta estacional semidecídua em Viçosa, Minas Gerais, e espécies de maior ocorrência na região. Revista Àrvore, Viçosa, v. 31, n. 6, p. 1121-1130, 2007.

GARCIA, F. C. P. Relações Sistemáticas e Fitogeografia do gênero Inga Miller (Leguminosae, Mimosoideae, Ingeae) nas Florestas da Costa Sul e Sudeste do Brasil. 1998. 248 f. Tese (Doutorado em Biologia Vegetal) Universidade Estadual Paulista, Rio Claro, 1998.

ILDIS. Ildis World Database of Legumes (version 10.01). Disponível em: <http://www.ildis.org.> Acesso em: 28 nov. 2006.

ISELY, D. Native \& naturalized Leg. of the USA. Utah: Brigham Young University, 1998. 424 p.

JESUS, R. M.; GARCIA, A. O Herbário da Reserva Florestal de Linhares. In: CONGRESSO NACIONAL SOBRE ESSÊNCIAS NATIVAS, 2., 1992, São Paulo. Anais... São Paulo: Instituto Florestal, 1992. v. 4, p. 339-346.

JOLY, C. A. et al. Evolution of the Brazilian phytogeography classification systems: implications for biodiversity conservation. Ciência e Cultura, Campinas, v. 51, n. 5-6, p. 331-48, 1999.

KÖPPEN, W. Climatologia: com um estúdio de los climas de la tierra. México: Fundo de Cultura Econômica, 1948. 478 p.

LEOGIER, A. H. Desc. Flora Puerto Rico \& Adjacent Is-Spermatophyta 2. Puerto Rico: Editorial UPR, 1988. 608 p.

LEWIS, G. P. Legumes of Bahia. Kew: Royal Botanic Gardens, 1987. 369 p.

LEWIS, G. P. et al. Legumes of the world Royal
Botanic Gardens. Kew: Royal Botanic Gardens, 2005. $577 \mathrm{p}$.

LIMA, H. C. Leguminosas arbóreas da Mata Atlântica: uma análise da riqueza, padrões de distribuição geográfica e similaridades florísticas em remanescentes florestais do estado do Rio de Janeiro. 2000. 151 f. Tese (Doutorado em Ecologia) - Universidade Federal do Rio de Janeiro, Rio de Janeiro, 2000.

LIMA, H. C.; GUEDES-BRUNI, R. R. Serra de Macaé de Cima: Diversidade Florística e Conservação em Mata Atlântica. Rio de Janeiro: Instituto de Pesquisas Jardim Botânico do Rio de Janeiro, 1997. 346 p.

LOMBARDI, J. A.; GONÇALVES, M. Composição florística de dois remanescentes de Mata Atlântica do sudeste de Minas Gerais, Brasil. Revista Brasileira de Botânica, São Paulo, v. 23, n. 3, p. 255-282, 2000.

LOPES, W. P. et al. Estrutura fitossociológica de um trecho de vegetação arbórea no Parque Estadual do Rio Doce - Minas Gerais, Brasil. Acta Botânica Brasilica, Porto Alegre, v. 16, n. 4, p. 443-456, 2002.

LORENZI, H. Árvores Brasileiras: manual de identificação e cultivo de plantas arbóreas nativas do Brasil. Nova Odess: Plantarum, 1992. v. 1-2.

MARANGON, L. C. et al. Florística arbórea da Mata da Pedreira, município de Viçosa, Minas Gerais. Revista Árvore, Viçosa, v. 27, n. 2, p. 207-215, 2003.

MARTINS, E. M. O. Stryphynodendron Mart. (Leguminosae: Mimosoideae) com especial referência aos taxa amazônicos. Acta Amazônica, Manaus, v. 10-11, p. 3-100, 1981.

MEIRA-NETO, J. A. A.; MARTINS, F. R. Composição florística de uma floresta estacional montana no município de Viçosa- MG. Revista Árvore, Viçosa, v. 26, n. 4, p. 437-446, 2002.

MENDONÇA FILHO, C. V. Braúna, angico, jacarandá e outras leguminosas de Mata Atlântica: Estação Biológica de Caratinga, Minas Gerais. Belo Horizonte: Fundação Biodiversitas, 1996. $55 \mathrm{p}$.

MENDONÇA FILHO, R. C. et al. Flora vascular do cerrado. In: SANO, S. M. et al. (Eds.). Cerrado: ambiente e flora. Planaltina: Embrapa-CPAC, 1998. p. 289-539.

MORIM, M. P. Leguminosae arbustivas e arbóreas da Floresta Atlântica do Parque Nacional do Itatiaia, sudeste do Brasil: padrões de distribuição. Rodriguésia, Rio de Janeiro, v. 57, n. 1, 
p. 27-45, 2006.

NUNES, S. R. D. F. S. As Mimosoideae (Leguminosae) no Parque Estadual do Rio Doce, Minas Gerais, Brasil. 2003. 106 f. Dissertação (Mestrado em Botânica) - Universidade Federal de Viçosa, Viçosa, 2003.

NUNES, S. R. D. F. S. et al. Mimosoideae (Leguminosae) Arbóreas do Parque Estadual do Rio Doce, Minas Gerais, Brasil: distribuição geográfica e similaridade florística na Floresta Atlântica no Sudeste do Brasil. Rodriguésia, Rio de Janeiro, v. 58, n. 2, p. 403-421, 2007.

OLIVEIRA-FILHO, A. T. et al. Camparison of the woody flora and soils of six areas of montane semideciduous Forest in southern Minas Gerais, Brazil. Edinburgh Journal of Botany, Edinburgh, v. 51, n. 3, p. 335-389, 1994.

OLIVEIRA-FILHO, A. T.; FONTES, M. A. L. Patterns of floristic differentiation among Atlantic Forests in Southeastern Brazil and the influence of climate. Biotropica, Washington, v. 32, n. 4b, p. 793-810, 2000.

OLIVEIRA-FILHO, A. T.; MACHADO, J. N. M. Composição florística de uma floresta semidecídua submontana, na serra de São José, Tiradentes, Minas Gerais. Acta Botânica Brasílica, Porto Alegre, v. 7, n. 2, p. 107-118, 1993.

PENNINGTON, T. D. The genus Inga: Botany. Kew: Royal Botanic Gardens, 1997. 844 p.

POLHILL, R. M.; RAVEN, P. H. Advances in legume systematics. Kew: Royal Botanic Gardens, 1981. v. 1-2

PRADO, D. E.; GIBBS, P. E. Patterns of species distributions in dry seasonal forests of South America. Annals of Missouri Botanical Garden, St. Louis, v. 80, p. 902-927, 1993.

RODRIGUES, L. A. et al. Florística e Estrutura da comunidade arbórea de um fragmento florestal de Luminária, MG. Acta Botânica Brasílica, Porto Alegre, v. 17, n. 1, p. 71-87, 2003.

SEVILHA, A. C. et al. Fitossociologia do estrato arbóreo de um trecho de floresta estacional no Jardim Botânico da Universidade Federal de Viçosa (face sudoeste), Viçosa, Minas Gerais. Revista Árvore, Viçosa, v. 25, n. 4, p. 431-443, 2001. SILVA, A. C. A. As Mimosoideae (Leguminosae) de um Fragmento de Floresta Estacional Semidecídua Montana, Região do Manso, Antônio Dias, MG. 2006. 55 f. Monografia (Graduaçao em Ciências Biológicas) - Centro Universitário do Leste de Minas Gerais, Ipatinga, 2006.

SILVA, L. Á.; SOARES, J. J. Composição florística de um fragmento de floresta estacional semidecídua no município de São Carlos-SP. Revista Árvore, Viçosa, v. 27, n. 5, p. 647-656, 2003.

SNEATH, P. H. A.; SOKAL, R. R. Numerical Taxonomy. San Francisco: W. H. Freeman, 1973. $345 \mathrm{p}$.

SOARES, M. P. et al. Composição florística do estrato arbóreo de uma floresta atlântica interiorana em Araponga - Minas Gerais. Revista Árvore, Viçosa, v. 30, n. 5, p. 859-870, 2006.

SOUZA, V. C.; LORENZI, H. Botânica Sistemática: guia ilustrado para identificação das famílias de fanerógamas nativas e exóticas no Brasil, baseado em APG II. 2. ed. Nova Odessa: Instituto Plantarum, 2008. 640 p.

VELOSO, H. P. et al. Classificação da vegetação brasileira, adaptada a um sistema universal. Rio de Janeiro: IBGE, 1991. 124 p.

WOJCIECHOWSKI, M. F. Reconstructing the phylogeny of legumes (Leguminosae): an early $21 \mathrm{st}$ century perspective. In: KLITGAARD, B. B. et al. (Eds.). Advances in Legume Systematics. Part 10. Kew: Royal Botanic Gardens, 2003. p. 5-35.

WOJCIECHOWSKI, M. F. et al. J. A phylogeny of Legumes (Leguminosae) basead on analysis of the plastid mat-K gene resolves many well-supported subclades within the family. American Journal of Botany, St. Louis, v. 91, n. 11, p. 1846-1862, 2004. YAMAMOTO, L.F. etal. Florística dos componentes arbóreo e arbustivos de um trecho da Floresta Estacional Montana, município de Pedreira, estado de São Paulo. Revista Brasileira de Botânica, São Paulo, v. 28, n. 1, p. 191-202, 2005. 